\title{
Erratum to: Variants of EVER1 and EVER2 (TMC6 and TMC8) and human papillomavirus status in patients with mucosal squamous cell carcinoma of the head and neck
}

\author{
Annika Antonsson ${ }^{1}$ Matthew H. Law ${ }^{2}$ Rachel E. Neale ${ }^{1} \cdot$ William B. Coman ${ }^{3}$. \\ David I. Pryor ${ }^{3} \cdot$ Study of Digestive Health (SDH) - Sandro V. Porceddu ${ }^{3}$. \\ David C. Whiteman ${ }^{1}$
}

Published online: 9 June 2016

(C) Springer International Publishing Switzerland 2016

\section{Erratum to: Cancer Causes Control (2016) 27:809-815 DOI 10.1007/s10552-016-0749-y}

We have wrongly stated that the SNP rs7205422 was analyzed instead of the EVER SNP rs7208422 in two places in this publication, namely the abstract and in the
"Results" section (para SNP analysis, 2nd line). Otherwise, rs7208422 has been correctly used throughout the paper.

The authors would like to apologize for any inconvenience caused.

The online version of the original article can be found under doi:10.1007/s10552-016-0749-y.

Annika Antonsson

Annika.Antonsson@qimrberghofer.edu.au

1 Department of Population Health, QIMR Berghofer Medical Research Institute, 300 Herston Road, Herston, QLD 4006, Australia

2 Statistical Genetics, QIMR Berghofer Medical Research Institute, Brisbane, QLD, Australia

3 Princess Alexandra Hospital, Brisbane, QLD, Australia 